\title{
DATA REDUCTION OF CRISM DATA TO HIGHLIGHT ALTERATION MINERALS
}

\author{
B. Bultel ${ }^{1}$, C. Quantin ${ }^{1}$, M. Andreani ${ }^{1}$, H. Clenet ${ }^{1,2}$ \\ ${ }^{1}$ Laboratoire de Géologie de Lyon (Laboratoire de Géologie de Lyon UCBL/ENS Lyon, France. \\ ${ }^{2}$ ICMP/EPSL École Polytechnique Fédérale de Lausanne, Switzerland.
}

\begin{abstract}
CRISM (Compact Reconnaissance Imaging Spectrometer for Mars) hyperspectral data have a spatial resolution ranging from 12 to $36 \mathrm{~m} /$ pixel allowing the high resolution mapping of minerals at the surface of Mars. However, the signal-to-noise ratio (SNR) makes challenging the discrimination of minerals spectrally close such as certain phyllosilicates and carbonates. Here, we discuss different processing of data reduction used to improve the signal-to-noise ratio and to highlight the alteration minerals at the surface of Mars and their limit. We show that our tool allows to understand trends in global mineralogy present in hyperspectral data cube.
\end{abstract}

\section{INTRODUCTION}

CRISM onboard MRO (Mars Reconnaissance Orbiter) is a visible to near-infrared hyperspectral imaging spectrometer between $0.4-4.0 \mu \mathrm{m}$ with a spectral resolution of $6.10^{-3} \mu \mathrm{m}$. We here use the Targeted Data mode that acquires data at spatial resolutions ranging from 12 to $36 \mathrm{~m} / \mathrm{pixel}$. The $\mathrm{S}$ detector samples wavelengths from 0.4 to $1.0 \mu \mathrm{m}$ (107 channels), and the $\mathrm{L}$ detector samples wavelengths from 1.0 to $4.0 \mu \mathrm{m}$ (438 channels) [1]. The NIR domain is often used to detect the hydrated minerals on the surface as they have characteristic absorptions between $1.9 \mu \mathrm{m}$ and $2.6 \mu \mathrm{m}$ [1].

CAT (CRISM Analysis Toolkit) is a pipeline developed by the CRISM Team that allows the correction of the photometric effects, the atmosphere contribution and the first order noise [1, 2, 3 and 4]. Even after those processing, it is still challenging to discriminate different minerals with slight spectral difference like alteration mineral such as dolomite, serpentine and chlorite, [5, 6 and 7]. We present a processing of data reduction to use as a complement of CAT to remove the noise still present in the data after the use of CAT. We present here this method and its tests on synthetic

The research leading to these results has received funding from the European Research Council under the European Union's Seventh Framework Program (FP7/2007-2013)/ERC Grant agreement $\mathrm{n}^{\circ} 280168$. data cube built with artificially noised spectra from spectral library.

\section{METHOD}

\subsection{Preprocessing with CAT}

We use CAT to preprocess the CRISM data. It first applies a photometric correction [1]. The martian surface being consider as lambertian because of the high roughness and low reflectance of the martian regolith [8], the raw values are divided by the cosine of the incidence angle as a simple photometric correction to provide an approximation of reflectance. Then, it corrects contribution of the atmosphere [2 and 3], the atmospheric contribution being assessed by comparison between spectra acquired at the base and at the summit of Olympus Mons. CAT also includes a filtering tool (CIRRUS) that first remove the outlying values along the spectral dimension named as spectral spike by replacing the value by the median of neighbor values [4]. The filtering tool also removes the spatial stripes by the median of neighbor values in the spatial dimension. Even after these processing, data are still noisy especially the ones acquired after several years around Mars. Also, there is often a remaining spectral contribution of the Martian atmosphere.

\subsection{Spectral denoising}

We design a noise removal pipeline to be used after CAT preprocessing to denoised and smooth the data. It consists in the application of three successive filters: the sharpeningmedian filter, a mobile median and a mobile average filter. The sharpening-median filter detects outlying values by comparison between the reflectance at spectral channel $n$ and the nearby values included between $n-5$ and $n+5$. The values are sorted in ascending order in a matrix $T$. Two fences are defined for the lower limit (LwL) and upper limit (UpL) as follow:

$$
\begin{aligned}
& L w L=L w Q-1.5 \times \operatorname{In} Q \\
& U p L=U p Q+1.5 \times \operatorname{In} Q
\end{aligned}
$$


Where LwQ, InQ and UpQ are respectively the lower quartile, the inner quartile and the upper quartile. Then the outlying values are replaced by the median on a window of 5 values $(n-2$ to $n+2)$. The next filter is a mobile median used to smooth the data on a window of 5 values $(n=5)$. Finally, a mobile average $(n=5)$ is applied. The mobile average is sensible to the isolated values and spikes, the sharpeningmedian is so used to remove those values and replace them by the closest kept values and the mobile median is used to smooth the remaining spikes [5].

\subsection{Ratioing methods}

The remnant contribution of atmosphere, the presence of dust and the existence of instrumental artifacts still bother the study of any CRISM cube. Ratioing methods are used to remove their contributions [ 1 and 6]. The most usual method is to use as denominator a spectrum judged by the user as spectrally neutral when possible in the same column of detector as the target spectrum [6 and 9]. This method has the disadvantage to be manual and to possibly introduce a bias. We investigate here different way of automatic ratioing method of a CRISM data cube. First, we analyze the use of the median spectrum of the whole data cube as denominator. Then, for comparison, we process to the automatic ratio of the data cube by the median spectrum of each column that is supposed to remove the detector dependent effects, or the smile effect [1]. In some case, the median spectrum on each column is not spectrally neutral. We so developed an automatic tool to search for the most spectrally neutral spectrum of each column of detector. We call the processing further in the paper the "selective ratioing method". On each column, the spectrum with the less variation of reflectance channel by channel is selected to be the denominator for the column.

Figure 1 shows the results of the different ratioing techniques for a pixel of the CRISM data cube frt0000862b centered on a central peak of a crater in Tyrrhena Terra (Mars) where the possible present mineral phases are olivine, pyroxene, phyllosilicates and carbonates. First, we observe that for the 4 types of ratio, the remnant contribution of the Martian atmosphere centered at $2 \mu \mathrm{m}$ is removed. The combination of absorptions near $1.9 \mu \mathrm{m}, 2.3$ and $2.5 \mu \mathrm{m}$ are present whatever the ratio. This absorption combination is diagnostic of certain phyllosilicates and carbonates $[10,11$, $12,13,14$ and 15]. The global shape of the spectrum is different in the case of the selective ratio. The absorption near $1 \mu \mathrm{m}$ due to $\mathrm{Fe}^{2+}$ electronic transition $[10,10]$ is deeper, whereas the absorption near 2.7-2.9 $\mu \mathrm{m}[10,11]$ causing the drop after $2.2 \mu \mathrm{m}$ is not present on this spectrum. The artifact near $1.2 \mu \mathrm{m}$ is only present on the median global ratio. The artifact near $1.6 \mu \mathrm{m}$ is only present on the manual ratio and possibly on the median global ratio (flat). A comparison of the different ratios allows us to conclude that they are all efficient to highlight alteration minerals but that their use for interpretation of the global shape of the spectrum may by strongly affected by the shape of the denominator [1] while it may be more challenging for mafic mineral that have efficient way to highlight the surface.

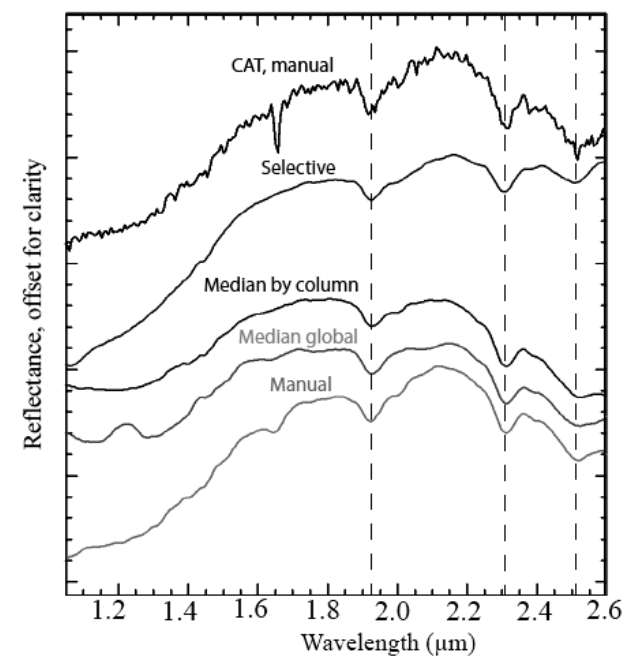

Figure 1: Result of the different ratioing methods applied to frt0000862b. The dotted lines indicate $1.92 \mu \mathrm{m}, 2.305 \mu \mathrm{m}$ and $2.51 \mu \mathrm{m}$, the spectrum obtain after CAT processing with a manual ratio is shown for comparison (CAT, manual).

\subsection{The 2.3-2.5 $\mu \mathrm{m}$ combination of absorption tools}

Alteration minerals such as phyllosilicates and carbonates have diagnostic combinations of absorptions near 2.3$2.5 \mu \mathrm{m}$. In first approximation, it is possible to discriminate them by determining the position of the center of those absorptions after removing the continuum because the combinations of center of absorption are different for phyllosilicates and carbonates as show in the figure $2[12$, 13,14 and 15]. We developed a tool that first detects and counts the pixels in the cube containing their main absorption near $2.3-2.5 \mu \mathrm{m}$, removes the continuum and determines the center of the absorption. Then, the number of pixels is reported on a graphic combining the position of the center of absorption near $2.3 \mu \mathrm{m}$ versus the position of center of absorption near $2.5 \mu \mathrm{m}$ (Figure 2).

The continuum is defined by an equation of a straight line between the shoulders of the absorption as follow:

$$
\begin{aligned}
& R_{C}=a \times \lambda_{C}+b \\
& a=\frac{R(r \lambda)-R(l \lambda)}{\lambda_{r \lambda}-\lambda_{l \lambda}}
\end{aligned}
$$




$$
b=R(l \lambda)-a \lambda_{l \lambda}
$$

Where $R(r \lambda)$ is the reflectance at the right shoulder, $R(1 \lambda)$ the reflectance the left shoulder, $\lambda_{\mathrm{r} \lambda}$ the wavelength at the right shoulder and $\lambda_{1 \lambda}$ the wavelength at the left shoulder. Our process is developed to be run systematically on a complete cube. The expression of the continuum removal is:

$$
R_{\text {final }}=\frac{R_{\text {data }}}{R_{C}}
$$

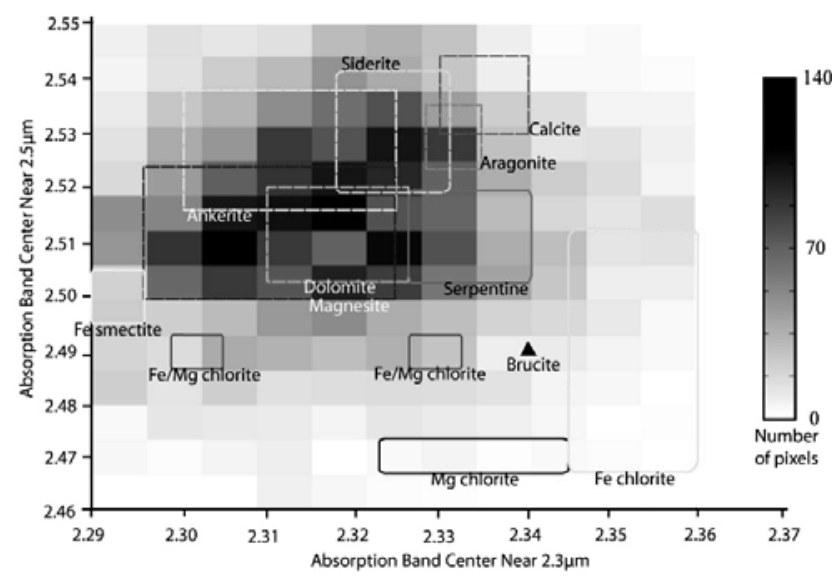

Figure 2: Position of the center of absorption near $2.3 \mu \mathrm{m}$ versus the position of the center of the absorption near $2.5 \mu \mathrm{m}$ for a CRISM data cube (frt000862b).

It allows us to understand trends in global mineralogy present in the cube studied. This tool is not claiming to conclude on the mineralogy present in a data cube but is a powerful guideline for the spectral analysis. In the figure 2, the dotted rectangles are the carbonates and the rectangles with full lines are the phyllosilicates. In this example, the dominant mineralogy seem to be carbonates more than phyllosilicates.

\section{TESTS}

\subsection{Test on the denoising method}

To test our spectral denoising tool, we generate synthetic data cubes from laboratory spectra. We use laboratory spectra from RELAB library. We then artificially introduce noise to our synthetic data cube to reach a similar signal-tonoise ratio (SNR) as CRISM data (around 100 [1]). We define the noise to add by normally-distributed (Gaussian) pseudo-random numbers with a mean of zero and a standard deviation of one. The SNR is estimated by calculating the ratio of mean to standard deviation of the signal [16]. We applied our personal pipeline to the noised spectrum and compare the result to the original spectrum. The goal is to test if absorptions features close to the noise level are modified or even erased by the spectral denoising pipeline. Figure 3 presents the case of a laboratory spectrum of serpentine artificially noised. The thin absorption of serpentine spectrum centered at $2.32 \mu \mathrm{m}$ is conserved at the same position but is slightly enlarged by the cleaning process. The absorptions smaller than the noise level are lost. The others are reduced by the smoothing steps and enlarged by the whole process but we still easily identify them with confidence until SNR is higher than 20. We conclude from these tests that our personal noise removal pipeline can be used for a SNR higher than 20 (CRISM SNR is supposed to be higher than 100 at all wavelength [1]).

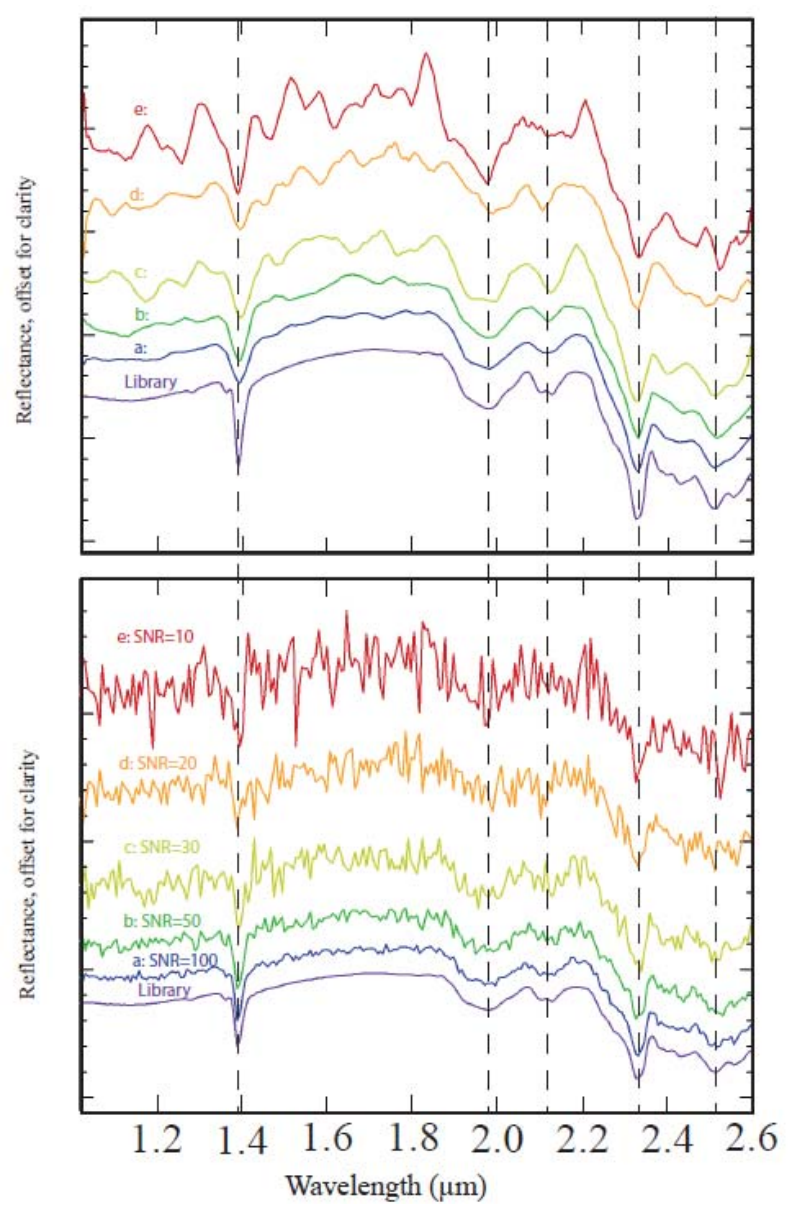

Figure 3: Lower: library spectrum of serpentine (from RELAB: SERPENTINE C2CR01). The library spectrum is noised with a signal-to-noise ratio of 100, 50, 30, 20 and 10. Upper: Result of our spectral denoising pipeline. The letters correspond to the letters of the noised spectra. The vertical dashed lines, showing the position of the diagnostic features, occur at $1.39 \mu \mathrm{m} ; 1.98 \mu \mathrm{m}$; $2.1 \mu \mathrm{m} ; 2.32 \mu \mathrm{m}$ and $2.51 \mu \mathrm{m}$. 


\subsection{Test of the $2.3-2.5 \mu m$ combination of absorption Tools}

We test the tool developed to highlight the $2.3-2.5 \mu \mathrm{m}$ combination of absorption on synthetic hyperspectral cube built from library spectra. The synthetic hyperspectral cube presented in figure 4 has 10,000 pixels with 100 pixels of dolomite, 100 of a Fe-rich chlorite and 100 of a Fe/Mg-rich chlorite. Others pixels correspond to fayalite, forsterite, diopside or enstatite (mafic minerals without any absorption near 2.3 and $2.5 \mu \mathrm{m}$ ). The dolomite and the Fe-rich chlorite are detected in the predicted area on the graphic. The Fe/Mg rich is at proximity of the area for a 50-50 Fe/Mg-chlorite. The robustness of the tool seems to be validated.

\section{CONCLUSION}

We demonstrate the efficiency of our spectral denoising pipeline on CRISM data and the robustness of our tool to discriminate minerals with spectral differences. Our analysis tools can be used for systematic study of the CRISM data to improve mineral detection and determination. Perspectives of development include the adaptation to others hyperspectral data and discrimination of phases other than phyllosilicates and carbonates.

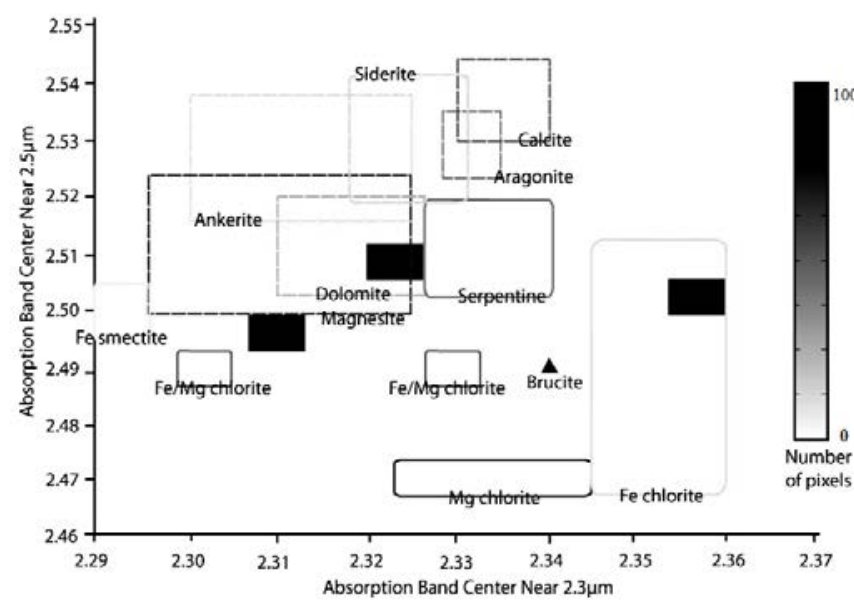

Figure 4: Position of the center of absorption near $2.3 \mu \mathrm{m}$ versus the position of the center of the absorption near $2.5 \mu \mathrm{m}$ for the synthetic data cube.

\section{REFERENCES}

[1] Murchie, S., et al. (2007), Compact Reconnaissance Imaging Spectrometer for Mars (CRISM) on Mars Reconnaissance Orbiter (MRO), J. Geophys. Res., 112, E05S03.

[2] Langevin et al., 2005b, Summer Evolution of the North Polar Cap of Mars as Observed by OMEGA/Mars Express, Science, Vol. 307, p. 1581-1584.
[3] McGuire et al., 2008, An improvement to the volcano-scan algorithm for atmospheric correction of CRISM and OMEGA spectral data, Transactions on geoscience and remote sensing, Vol. 46 Issue 12 p. 4020-4040.

[4] Parente, M., 2008, Denoising CRISM images: a new look, LPSC 39, \#1391.

[5] D'Agostino, R. B. and Stephens, M. A., 1986. Goodness-of-Fit Techniques, Marcel Dekker, Inc., New York. Chapter 12

[6] Ehlmann, B. L., et al. (2009), Identification of hydrated silicate minerals on Mars using MRO-CRISM: Geologic context near Nili Fossae and implications for aqueous alteration, J. Geophys. Res., 114.

[7] Bultel et al., 2013, A new CRISM data analysis tool for the detection of miscellaneous alteration phases, EPSC2013

[8] Johnson, J.R. et al., 2006. Spectrophotometric properties of materials observed by Pancam on the Mars Exploration Rovers: 1. Spirit. J. Geophys. Res. Planets, Vol. 111, E02S14.

[9] Carter, J. et al., (2013), Hydrous minerals on Mars as seen by the CRISM and OMEGA imaging spectrometers: Updated global view, J. Geophys. Res. Planets, 118.

[10] Salisbury, et al., 1991b, Infrared (2.1- 25 micrometers) Spectra of Minerals, Johns Hopkins University Press, 294 pp.

[11] Clark et al., 1990, High Spectral Resolution Reflectance Spectroscopy of Minerals, J. of Geophys. Res., Vol. 95, No. B8, Pages 12,653-12,680.

[12] Gaffey, 1987, Spectral reflectance of carbonate minerals in the visible and near infrared (0.35-2.55 um): anhydrous carbonate minerals, J. Geophys. Res., Vol. 92, No. B2, 1429-1440.

[13] Bishop et al., 2013, Coordinated spectral and XRD analyses of magnesite-nontronite-forsterite mixtures and implications for carbonates on Mars, J. Geophys. Res. Planets, Vol. 118, 487-513.

[14] Gaffey, 1986, Spectral reflectance of carbonate minerals and rocks in the visible and near infrared (0.35 - 2.55 microns) and its applications in carbonate petrology, American Mineralogist, Vol. 71, 151-162.

[15] Bishop et al., 2008, Reflectance and emission spectroscopy study of four groups of phyllosilicates: smectites, kaoliniteserpentines, chlorites and micas, Clay Minerals, 43, 35-54.

[16] Russ, J. C. (2010). The image processing handbook. CRC press Chapter 6. 